\title{
INVESTIGANDO O PROCESSAMENTO COGNITIVO DE TRADUTORES PROFISSIONAIS EM TRADUÇÃO DIRETA E INVERSA NO PAR LINGUÍSTICO INGLÊS- PORTUGUÊS
}

\author{
Aline Alves Ferreira \\ Universidade Federal de Minas Gerais \\ alineafe@gmail.com
}

Resumo: Este artigo investiga alguns aspectos cognitivos observados no desempenho de dez tradutores profissionais, na execução de tarefas envolvendo a tradução de textos correlatos da língua estrangeira (inglês) para a língua materna (português) - tradução direta (TD) - e da língua materna (português) para a língua estrangeira (inglês) - tradução inversa (TI). A coleta de dados segue a metodologia de triangulação (ALVES, 2001; 2003). A análise processual teve como base representações lineares obtidas por meio de gravações feitas com o programa Translog ${ }^{\odot}$, em conjunto com protocolos retrospectivos. Utilizou-se a direcionalidade como variável independente, tendo como base a pesquisa de Buchweitz e Alves (2006). Como variáveis dependentes de análise tem-se: (i) tempo despendido em cada fase (orientação, redação e revisão) e nas duas tarefas como um todo (JAKOBSEN, 2002; ALVES, 2005), (ii) recursividade (BUCHWEITZ \& ALVES, 2006) e (iii) segmentação processual (DRAGSTED, 2004; RODRIGUES, 2009). Os resultados indicam algumas tendências sobre o desempenho de todo o grupo, mas apontam para o caráter idiossincrático do processamento de cada sujeito que, a partir de uma complexa rede de conhecimentos e habilidades, trabalha no intuito de produzir um texto de chegada de acordo com cada tarefa proposta.

Palavras-chave: processo tradutório, direcionalidade em tradução, conhecimento experto em tradução. 


\title{
INVESTIGATING THE COGNITIVE PROCESSING OF PROFESSIONAL PORTUGUESE-ENGLISH TRANSLATORS IN DIRECT AND INVERSE TRANSLATION
}

\begin{abstract}
This paper investigates the cognitive aspects observed in the translation process of ten professional translators while translating two correlated texts from a foreign language (English) into a native language (Portuguese) - direct translation - and from the mother tongue (Portuguese) into a foreign language (English) - inverse translation. Data collection was based on the triangulation method (ALVES, 2001; 2003). The analysis of translation process data drew on linear representations generated by means of keylogged files recorded with the software Translog and analyzed in conjunction with retrospective protocols. Directionality of the translation task was the independent variable in the study which partially replicates the work of Buchweitz and Alves (2006). The dependent variables are: (i) time spent on each phase of the translation process (orientation, drafting, and revision) and on the two tasks (JAKOBSEN, 2002; ALVES, 2005), (ii) recursiveness (BUCHWEITZ \& ALVES, 2006), and (iii) segmentation (DRAGSTED, 2004; RODRIGUES, 2009). The analysis reveals some tendencies about the group. However, it is possible to point out the idiosyncratic traits observable in the output of each subject who, drawing on a complex set of knowledge and abilities, strives to produce target texts in compliance with the specificities of the translation task.
\end{abstract}

Keywords: translation process, directionality in translation, expert knowledge in translation.

\section{Introdução}

Nas últimas décadas, percebe-se um aumento no número de investigações sobre o processo tradutório a partir do uso de softwares capazes de monitorarem todo o processo de produção do texto de chegada, tais como Translog ${ }^{\odot}$ e Tobii Studio, entre outros. Neste contexto, os trabalhos que objetivam a identificação e o mapeamento do desempenho de tradutores a partir de uma abordagem processual têm apresentado grandes contribuições para os Estudos 
da Tradução. Contudo, a variável direcionalidade linguística ainda tem sido pouco investigada. Algumas das poucas discussões a respeito da prática da tradução inversa (TI, doravante) é levantada por Lorenzo (2003: 93), quando a autora aponta que os desafios apresentados pela TI se diferenciam mais em grau que em essência que os desafios relacionados às outras atividades tradutórias, já que os problemas relacionados à TI seriam os mesmos relacionados às outras tarefas de tradução: a diferença é que, segundo a autora, os problemas nas traduções inversas põem à prova a competência tradutória. Já Kelly et al. (2003) se mostram mais preocupados com a prática da TI em contraposição à da tradução direta (TD, doravante) quando, segundo os autores, a própria terminologia já indica uma relação hierárquica: uma é subordinada à outra, e a tradução inversa se mostra como um processo contrário ao normal, ao esperado e ao legítimo.

Buchweitz e Alves (2006) realizaram uma pesquisa de cunho empírico-experimental no intuito de verificar a influência da direcionalidade sobre o desempenho de dois grupos diferentes, sendo que um deles apresentava alguma experiência em tradução e o outro era formado por alunos do curso de especialização em tradução da UFMG. A pesquisa apresentou três objetivos principais, a saber: a) acessar qualitativamente todo o processo de produção do texto alvo; b) combinar os dados quantitativos e os dados qualitativos para descrever a produção desse texto e c) medir a recursividade ocorrida durante o processo de tradução. Os dados obtidos do processo tradutório foram correlacionados às informações oriundas dos relatos retrospectivos, usados no intuito de permitir aos pesquisadores fazerem inferências a respeito dos processos cognitivos em diferentes situações de tomada de decisão. A pesquisa dos autores apresentou grandes contribuições para os estudos sobre a direcionalidade linguística no processo de tradução, mas a análise foi feita a partir dos dados oriundos de diferentes perfis de tradutores. Nesse sentido, o presente artigo replica o experimento de Buchweitz e Alves (2006), sendo que a análise será pautada nos dados processuais observados 
no desempenho de dez tradutores profissionais, enfocando o impacto da direcionalidade sobre o processamento cognitivo desses sujeitos e, ainda, será verificada a ocorrência de pausas em cada uma das três fases do processo tradutório (orientação, redação e revisão final) na tradução de textos correlatos do inglês para o português (TD) e do português para o inglês (TI). Dessa maneira, esta pesquisa apresenta as seguintes variáveis de análise: (i) tempo despendido em cada fase e nas duas tarefas como um todo (JAKOBSEN, 2002; ALVES, 2005), (ii) recursividade (BUCHWEITZ \& ALVES, 2006) e (iii) segmentação processual (DRAGSTED, 2004; RODRIGUES, 2009). Os tradutores realizaram duas tarefas de tradução (uma TD e uma TI) de textos correlatos. A pesquisa partiu da hipótese de que os tradutores mudariam de comportamento de acordo com cada tarefa, dependendo do esforço cognitivo exigido, conforme apontado em Buchweitz e Alves (2006). Os autores observaram que a direção inversa consumiu mais tempo e aumentou o número de segmentos em ambos os grupos. Por outro lado, o grupo mais experimente apresentou aumento no número de revisões feitas na tradução inversa, indicando que a recursividade pode ser uma característica relacionada ao desempenho de tradutores mais experientes. As mudanças podem ser verificadas, quantitativamente, a partir dos números concernentes às variáveis supracitadas e, qualitativamente, a partir dos relatos retrospectivos coletados após a realização de cada tarefa, considerando a ordem de realização das tarefas como fator decisivo nas análises.

\section{Fundamentação Teórica}

De maneira geral, a tradução inversa foi vista, ainda nos primeiros anos do auge dos estudos tradutórios, de forma extremamente negativa por parte dos pesquisadores. Vários são os autores que defendem o exercício da tradução apenas para sua língua materna (BENJAMIN, 1982; BARNSTONE, 1993; NEWMARK, 1987), ainda que os conceitos língua materna e falante nativo não 
apresentem definições suficientemente claras, já que os critérios encontrados na linguística para tais definições não são capazes de precisá-las completa e objetivamente (POKORN, 2005). Dessa forma, a inexatidão do termo pode variar dependendo da intenção de uso da palavra. Um árduo crítico do exercício da TI foi Jean-René Ladmiral (1979), que negou inclusive sua utilidade como exercício pedagógico. Newmark (1987: 20) afirma que a tradução direta é a única forma de traduzir com naturalidade, exatidão e eficácia. $\mathrm{O}$ autor reconhece que a TI é executada em grande número, mas ao traduzir para outra língua que não a materna, o tradutor "é motivo de risos para muita gente ${ }^{1}$ ". Para o acadêmico Valentín García Yerba (1983) a TI se mostra quase impossível - a não ser em casos de bilinguismo total, e mesmo assim com certas limitações.

Poucos trabalhos sobre a influência da direcionalidade no processamento cognitivo de tradutores foram realizados até o presente momento. No Brasil, o único trabalho que investiga a variável foi a pesquisa realizada por Buchweitz e Alves (2006), mencionada anteriormente ${ }^{2}$. Os dados daquela pesquisa foram obtidos através do programa Translog ${ }^{\circledR}$, já que o software facilita a visualização da divisão do processo tradutório nos seus três estágios (orientação, redação e revisão) ${ }^{3}$. Tal divisão foi primeiro apontada por Jakobsen (2002) e permite-nos analisar a contagem do tempo despendido em cada uma das três fases de forma apurada.

Os autores observaram que a maioria dos tradutores gastou mais tempo ao traduzir inversamente (do português para o inglês) que diretamente (do inglês para o português), possivelmente porque a TI exigiu mais esforços. Outros trabalhos já haviam apontado que a TI exige mais tempo por parte dos tradutores (KROLL \& STEWART, 1994; MILLER \& KROLL, 2002).

A análise da recursividade foi feita a partir da movimentação do sujeito no texto, realizada por meio de movimentos com o mouse (incluindo a seleção de partes do texto) ou no teclado (setas do cursor). Ela inclui, ainda, o acionamento das teclas de eliminação (delete e backspace), bem como os acionamentos de End, Home, 
PgUp e PgDn. Ainda a partir do programa Translog ${ }^{\circledR}$, Buchweitz e Alves (2006) apontaram que quase todos os sujeitos gastaram mais tempo ao traduzirem inversamente que diretamente, o que leva os autores à inferência de que a dificuldade pode ter sido um fator decisivo na contagem de tempo despendido. Analisando as fases de redação e revisão final separadamente, Buchweitz e Alves (2006) concluíram que o grupo mais experiente realizou mais movimentos recursivos durante a revisão final ${ }^{4}$.

Os pesquisadores também verificaram o principal nível de segmentação em que o sujeito produzia seu texto - morfema, palavra ou frase. Para Alves (2003) e Jakobsen (2003), a segmentação cognitiva é um indicador do nível no qual o sujeito opera. De acordo com Alves (2003), se o tradutor está trabalhando no nível da palavra, pode resultar-lhe difícil construir uma rede textual coerente. Dessa maneira, se o leitor verificar isoladamente os dados da segmentação apresentados em Buchweitz e Alves (2006), ele terá a impressão que o grupo mais experiente está concentrado em segmentos menores no texto. Ou seja, seu ritmo cognitivo (SCHILPEROORD, 1996; ALVES, 2003) se apresentaria mais errático, o que não condiz com uma análise mais ampla dos resultados. Os autores concluíram que o grupo mais experiente está, a todo momento, tentando construir uma rede textual, no intuito de criar um texto alvo mais sólido, o que é apontado pelo aumento da recursividade.

A combinação das técnicas usadas na pesquisa de Buchweitz e Alves (2006) serviu para melhor descrever as estratégias usadas pelos sujeitos para construir o texto de chegada. Tais estratégias somadas aos dados de segmentação e recursividade ajudam-nos a identificar o comportamento adaptativo dos sujeitos de acordo com o grau de dificuldade da tarefa. Dessa maneira, o presente trabalho replica o experimento realizado em Buchweitz e Alves (2006), buscando analisar as variáveis a partir de perfis semelhantes de tradutores (e.g. tradutores experientes), para então contrastar os resultados com aqueles obtidos no experimento de Buchweitz e Alves (2006), verificando se e em que medida a direção linguística 
afeta o desempenho do grupo com relação ao tempo despendido na realização das tarefas, a ocorrência de movimentos recursivos e a segmentação processual dos textos.

\section{Metodologia}

A metodologia utilizada no escopo do presente artigo é baseada na triangulação dos dados processuais, proposta por Alves (2001; 2003). Todos os tradutores participaram de uma tarefa de TD e outra de TI, de textos correlatos. Os tradutores, que apresentam um perfil similar, se submeteram a um questionário prospectivo a fim de que pudessem ser identificados enquanto sujeitos adequados ao experimento. Também tiveram acesso a um termo de consentimento, assinado após a leitura e conformidade. Pediu-se aos primeiros cinco sujeitos ( $\mathrm{S} 1 \mathrm{a}$ S5) que traduzissem primeiro o texto em inglês (TD) e que depois traduzissem o texto em português (TI). Para o segundo grupo, formado pelos outros cinco sujeitos (S6 a S10) pediu-se que fosse realizada primeiro a TI e depois a TD. A ordem de realização das traduções foi alterada aleatoriamente entre o grupo, a fim de evitar o efeito de familiaridade quando da realização da segunda tarefa. Após a realização de cada tradução, pediu-se aos sujeitos que verbalizassem sobre suas tomadas de decisão na produção do texto alvo, fazendo-se uso da função Replay do Trans$\log ^{\odot}$ e do rastreador ocular Tobii Studio T60

Para a realização das análises no escopo do presente trabalho, têm-se as seguintes variáveis dependentes: (i) tempo, (ii) segmentação e (iii) recursividade, a fim de aferir o nível de automonitoramento dos sujeitos. Já as variáveis controladas são: i) par linguístico (português-inglês/inglês-português), ii) perfil de sujeitos (tradutores profissionais), iii) texto-fonte ${ }^{6}$ e iv) tarefa tradutória (tradução visando à publicação do texto em periódico internacional), além das condições de tradução, quais sejam: sem pressão de tempo e em ambiente $\operatorname{Translog}{ }^{\odot}$. A variável independente é a direcionalidade (TD e TI). 
Como já mencionado, Buchweitz e Alves (2006) analisaram os dados a partir de dois grupos de perfis distintos no intuito de verificar as diferenças processuais nos dois grupos, principalmente aquelas concernentes às variáveis recursividade e segmentação. A partir desses dados, os autores verificaram que os tradutores mais experientes apresentam níveis mais altos de consciência crítica e instâncias de metarreflexão, corroborando trabalhos anteriores (ALVES, 2003; ALVES, 2005). Nesse sentido, a presente pesquisa busca analisar as mesmas instâncias a partir de um grupo de tradutores que apresentem um perfil semelhante, a fim de apresentar conclusões passíveis de generalização.

\section{Resultados e Análises}

Como mencionado anteriormente, a análise dos dados é feita a partir das representações lineares geradas pelo Translog ${ }^{\odot}$ e dos relatos retrospectivos. As representações ilustram o processo da tradução, mostrando, por meio de símbolos, as pausas iguais ou superiores a cinco segundos (definidas pelo pesquisador) e os movimentos de recursividade, como o acionamento de mouse, cursor e teclas de delete e backspace. Cada segmento é delimitado por duas pausas iguais ou superiores a cinco segundos. Para cada segmento, foi contabilizado o número de movimentos de recursividade. As representações do $\operatorname{Translog}^{\circledR}$ também permitem a identificação e o cálculo da duração total das fases de orientação, redação e revisão (Jakobsen, 2002), as quais foram identificadas em termos absolutos (segundos) e relativos (porcentagem em relação ao total de tempo despendido para a realização de toda a tarefa tradutória). Foi feita uma correlação entre as análises quantitativas e qualitativas (JAKOBSEN, 2002; ALVES, 2003a) dos diversos dados coletados (i.e. entrevistas prospectivas, representações lineares do Translog ${ }^{\odot}$ e relatos retrospectivos) no intuito de tentar delinear uma configuração do desempenho dos tradutores em foco. Os resultados a se- 
guir apontam para tendências observadas sobre o tempo total gasto na execução das tarefas e em cada uma das fases separadamente, sobre a quantificação dos movimentos recursivos e sobre o número e tipo dos segmentos.

\section{Tempo}

A análise intersubjetiva sobre as três fases indica que o grupo mostra um comportamento bastante variável. Quanto ao tempo total de produção, cinco sujeitos gastaram mais tempo durante as traduções diretas e cinco gastaram mais tempo quando das traduções inversas. Cumpre salientar que a ordem de execução das tarefas exerceu grande influência no desempenho dos sujeitos, resultando num efeito facilitador, de maneira que, quando a tradução direta foi realizada primeiro, apenas um tradutor gasta mais tempo quando da segunda tradução (TI).

Em média, os tradutores gastaram mais tempo na tradução inversa $(4100,3 s)$ que quando da tradução direta $(3666,1 s)$. Entretanto, deve-se considerar que um tradutor (S10) apresenta um comportamento bastante divergente em comparação aos outros sujeitos analisados. Se excluímos os dados de S10, vemos que a diferença entre as médias cai consideravelmente $(\mathrm{TD}=3530,3 \mathrm{~s}$ e $\mathrm{TI}=3551,7)$.

Percebe-se, contudo, que um importante fator que pode ter influenciado o tempo de dedicação às tarefas é a frequência de realização de traduções ao inglês ${ }^{7}$. O sujeito S1, por exemplo, verbaliza dois fatores relevantes para tal análise. Além de o tradutor afirmar realizar mais traduções inversas, há de se considerar que ele realizou a tarefa direta antes de realizar a tradução inversa, ou seja, a ordem de realização, assim como sua prática, teve um efeito facilitador quando da tradução inversa. Verificou-se, portanto, que a ordem de realização das tarefas deverá ser considerada e, dentro da ordem, serão considerados os tempos de redação e de revisão final despendido em cada direção. 
Ao observar apenas a fase de orientação inicial intersubjetivamente, percebe-se que os tradutores dedicam pouco tempo a essa fase. A maioria dos sujeitos despendeu menos de dois minutos na fase de orientação, nas duas direções.

Em termos absolutos, a diferença entre o tempo gasto em segundos na segunda fase variou sistematicamente, mantendo um certo equilíbrio entre a TD e a TI. A fase de redação foi aquela que exigiu mais tempo, corroborando os achados de outros pesquisadores (JENSEN, 2001; JAKOBSEN, 2002, 2003; LIPARINI CAMPOS, 2005). O tempo médio gasto nessa fase durante a TD foi 2504,5 segundos e 2275,5 segundos quando da TI. Durante a fase de revisão, os tradutores gastaram em média 1118,2 segundos quando da TD e 1635,3 segundos quando da TI. Entretanto, seis sujeitos gastaram mais tempo de redação durante a TI que enquanto traduziam diretamente (S5 ao S10). Observa-se que, quando a TD é realizada primeiro, o tempo despendido com a fase de redação nessa direção é normalmente maior que o tempo gasto na mesma fase durante a TI. Na fase de revisão final, os tradutores gastaram mais tempo de revisão nas traduções inversas. É importante lembrar que os tradutores gastaram, em média, mais tempo com a fase de redação durante a TD, o que levaria a uma possível diminuição no tempo médio gasto durante a fase de revisão na mesma direção.

\section{Recursividade}

No intuito de verificar se a variável direcionalidade tem efeito sobre a recursão durante o processo tradutório, serão apresentados dados que contabilizam o número de movimentos de mouse, de teclas de navegação e de eliminação, conforme apresentado em Buchweitz e Alves (2006). Os autores contabilizaram os movimentos por teclas de eliminação, como delete e backspace, de navega-

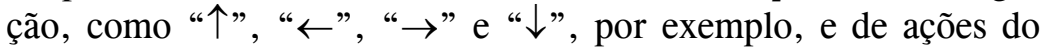
mouse, durante as fases de redação e revisão final separadamente. De acordo com a contagem total dos três tipos de movimentos e 
do número de teclas de produção e de eventos, observa-se maior número de acionamentos de teclas de eliminação (média $=200$ ), seguido por acionamentos de teclas de navegação (média $=151,4)$ e mouse (média $=127,9$ ) quando das traduções diretas. A média de acionamento de teclas de eliminação durante a TI é de 199, sendo praticamente a mesma observada durante a TD. Já as médias de movimentos de navegação e de mouse foram superiores quando da tradução inversa: 196,5 e 177,3, respectivamente. Verifica-se que os movimentos de recursão durante a TI totalizam 5868 acionamentos de teclas, número superior àquele encontrado na tradução direta (4793). Observando a fase de redação e de revisão final separadamente, conclui-se que, durante a fase de redação, a recursividade é menor durante a tradução direta: 14,99 acionamentos a cada 100 teclas. Quando da tradução inversa, verifica-se 18,21 acionamentos recursivos a cada 100 teclas. Esse resultado já era esperado visto que os tradutores realizaram mais movimentos recursivos na tradução inversa como um todo. Esses movimentos indicam que os tradutores, ao realizarem mudanças, correções e eliminações de trechos traduzidos estão, já na fase de redação, em constante revisão de sua produção textual, considerada por Jakobsen (2002) como revisão online. Já na fase de revisão final, durante a tradução direta verifica-se que a cada 100 eventos registrados, 54,06 foram movimentos recursivos. Quando da tradução inversa, a cada 100 teclas acionadas, 62,08 são teclas que indicam movimento recursivo (número superior ao verificado quando da revisão durante a TD). Tais dados corroboram a afirmação de Jakobsen (2002) sobre a característica recursiva e de menor produção textual da fase de revisão.

\section{Segmentação}

A análise segmental replica aquela realizada em Buchweitz e Alves (2006), no sentido de se considerar um segmento como produção textual realizada entre pausas de pelo menos cinco segundos. 
Porém, a classificação dos segmentos segue a classificação apresentada em Rodrigues (2009), que teve como base o trabalho de Dragsted (2004). Buchweitz e Alves (2006) observaram a segmentação dos tradutores nos níveis do morfema, palavra e sentença. Já Rodrigues (2009) divide os segmentos em seis distintas categorias, a saber: i) Segmentos Transentenciais (TS - são tipos especiais de segmentos Não Sintáticos que incluem elementos de mais de uma sentença); ii) Complexos Oracionais (CO - segmentos que envolvem mais de uma oração); iii) Oração ( $\mathrm{O}$ - constituintes que incluem pelo menos um elemento verbal e um sujeito, objeto direto, objeto indireto, complemento ou elemento indicando o início de uma oração subordinada); iv) Grupo/Sintagma (G - constituintes que incluem pelo menos duas palavras, sendo que uma delas pode ser apontada como o elemento central, identificado como o núcleo); v) Palavra ( $\mathrm{P}$ - qualquer sequência de caracteres entre dois espaçamentos) e vi) Segmentos Não Sintáticos (NS - segmentos não motivados sintaticamente).

Os dados da presente pesquisa apontam que não há relação entre a ordem de realização das tarefas e o número de segmentos, sendo que cada tradutor apresenta um padrão idiossincrático. Entretanto, considerando todo o grupo, percebe-se um aumento de segmentos quando da TI. De maneira geral, o grupo apresentou mais segmentos no nível do Grupo/Sintagma (G) e no nível da Palavra (P). Durante a TD, houve predomínio de segmentos no nível do Grupo/ Sintagma (G) na produção de sete sujeitos e os demais tradutores produziram mais segmentos no nível da Palavra (P). Quando da TI, seis tradutores apresentaram mais segmentos no nível da Palavra, três apresentaram maior número de segmentos no nível do Grupo/Sintagma (G) e um tradutor apresenta o mesmo número de segmentos para as duas categorias (Palavra e Grupo/Sintagma). O predomínio de segmentos nos níveis da Palavra e do Grupo/Sintagma é percebido tanto na tradução como um todo quanto quando se observam as fases de redação e de revisão final separadamente, revelando que os tradutores segmentaram os textos em ordens hie- 
rárquicas inferiores ${ }^{8}$. Ainda que não haja um padrão com relação à média de palavras por segmento, percebe-se um aumento quando da TD para a maioria dos tradutores ${ }^{9}$, revelando que a TI foi mais segmentada que a TD.

\section{Conclusão e desdobramentos}

Foram analisados o tempo gasto, o número e o tipo predominante de movimentos recursivos e o número e o tamanho dos segmentos produzidos durante as duas tarefas como um todo e em cada uma das três fases do processo tradutório especificamente. Logo após, contrastaram-se os dados com aqueles observados no trabalho de Buchweitz e Alves (2006).

Observa-se que os tradutores, em geral, despendem pouco tempo na fase de orientação inicial, corroborando os dados apontados em trabalhos anteriores (BUCHWEITZ \& ALVES, 2006; LIPARINI CAMPOS, 2010; RODRIGUES, 2010; ALVES \& LIPARINI CAMPOS, 2009). Os resultados referentes ao tempo dedicado à fase de redação indicam que a ordem de realização da tarefa teve mais impacto que a própria variável direcionalidade, diferentemente do que aconteceu no estudo de Buchweitz e Alves (2006), quando se verificou que a maioria dos sujeitos gastou mais tempo de redação durante a TI. Na presente pesquisa, observa-se que do grupo de tradutores que realizaram primeiro a TD, os quatro primeiros sujeitos gastaram mais tempo nessa direção que durante a TI, enquanto todo o segundo grupo, que realizou primeiro a TI, dedica mais tempo à fase de redação nessa direção. Contudo, percentualmente, a maior parte do grupo (sete tradutores) apresenta maior tempo despendido durante a fase de redação quando da TD que quando da TI. Com relação à fase de revisão final, percebe-se que os tradutores tendem a aumentar o tempo ao traduzirem inversamente, ao contrário do que foi observado em Buchweitz e Alves (2006). Analisando as duas tarefas e considerando a or- 
dem de realização, verifica-se uma tendência ao aumento de tempo quando da primeira tradução. Em média, constata-se que não há grande divergência entre o dispêndio de tempo nas duas direções, contrariando os dados apresentados em Buchweitz e Alves (2006), quando os pesquisadores observaram um aumento bastante relevante quando da TI.

A variável recursividade apresenta resultados que não estão relacionados à ordem de execução das tarefas, quando se observa um aumento nos três tipos de movimentos de recursão quando das traduções inversas. Apenas três tradutores apresentam maior recursividade durante a TD que quando da TI, o que indica maior retomada dos sujeitos a segmentos já traduzidos do português para $o$ inglês, tanto na fase de redação quanto na de revisão. Esses dados corroboram os resultados de Buchweitz e Alves (2006), que apontaram mais movimentos recursivos durante a tradução inversa de maneira geral, seja durante a revisão online ou quando da revisão final do produto tradutório.

No que concerne à segmentação, o grupo que realizou primeiramente a TD apresenta uma tendência a aumentar o número de segmentos quando da segunda tradução (TI). Já no grupo que realizou primeiro a TI, percebe-se uma tendência à diminuição de segmentos quando da segunda tradução (TD). Dessa maneira, percebe-se um aumento de segmentos durante as traduções inversas, conforme também apontado em Buchweitz e Alves (2006). Contudo, ao observar os números individualmente, percebe-se que cada sujeito apresenta um padrão de distribuição próprio e que não está atrelado à ordem de realização das tarefas. Com relação ao tipo de segmento, houve predomínio no nível do Grupo/Sintagma durante a TD, seguido pelo nível da Palavra. Durante a TI, ocorre uma inversão e observa-se predomínio no nível da Palavra, seguido pelo Grupo/ Sintagma. Ainda assim, os dados das duas tarefas indicam uma tendência para a segmentação em ordens hierárquicas inferiores. Contudo, não se verifica um padrão entre os sujeitos, sendo que o tamanho, tipo e a quantidade predominante de segmentos varia de acordo com o perfil do tradutor. 
É importante ressaltar que a análise segmental apresentada em Buchweitz e Alves (2006) foi relacionada com a análise da recursividade nos dados apresentados, sugerindo que um alto número de recursividade associado à grande quantidade de segmentos podem indicar adaptação à tarefa tradutória. Por outro lado, se não há aumento na recursividade mas há aumento no número de segmentos, isso é um indicativo de que o tradutor está lidando com um problema o qual ele não consegue resolver. Os autores constataram que o grupo mais experiente apresentou um aumento no número de segmentos acompanhado por um aumento no número de movimentos recursivos, indicando que o grupo está, de fato, se adaptando à tarefa proposta. Da mesma maneira, o grupo da presente pesquisa aumenta o número de movimentos recursivos quando da tradução inversa, que acompanha o aumento na quantidade de segmentos quando da mesma direção.

A presente pesquisa partiu da hipótese de que a direção linguística obrigaria os tradutores a modificarem seus processos no intuito de conseguirem finalizar a tradução de forma que o produto tradutório estivesse de acordo com o Brief apresentado antes do início das tarefas. Contudo, ressalta-se que, no escopo do presente artigo, mais importante que a análise da variável direcionalidade é a análise da ordem de execução da tarefa tradutória. Optou-se então por separar os tradutores em dois grupos - S1 a S5 (que realiza a TD primeiro) e S6 a S10 (que realiza a TI primeiro), e então verificar em que medida a direção linguística afeta o desempenho do grupo, conforme apresentado previamente. Foi observado que os tradutores tendem a realizar a segunda tarefa de maneira mais otimizada ao aproveitarem o conhecimento adquirido durante a primeira tradução, tratando-se de textos de partida correlatos ${ }^{10}$. Contudo, os resultados sobre a recursividade e a segmentação não apontam para uma estrita correlação entre essas variáveis e a ordem de realização das tarefas, como observado nas variáveis tempo e pausa ${ }^{11}$. Mais especificamente, a recursividade mostra-se como medida de adaptação cognitiva quando da realização de traduções inversas, corroborando os resultados encontrados em Buchweitz e Alves (2006). 
Tendo em vista a importância e a forte ocorrência de tarefas de tradução inversa para a prática profissional de tradutores experientes, considera-se de grande valia estudos continuados que tratem a temática e insiram outras ferramentas de análise. Ainda, considera-se que a replicação da metodologia poderá gerar resultados comparáveis aos aqui apresentados e assim desenvolver conceitos sobre o processo tradutório passíveis de generalização no campo dos Estudos da Tradução.

Recebido em 20/07/11

Aceito em 11/10/11

\section{Notas}

1. Citação original: "In fact, however, most translators do translate out of their own language ('service' translation) and contribute greatly to many people's hilarity in the process".

2. Como texto de partida, Buchweitz e Alves (2006) selecionaram dois artigos jornalísticos sobre o envio de soldados brasileiros ao Haiti. O critério de seleção para a escolha dos textos foi o tamanho e o prestígio dos periódicos nos quais os textos foram publicados. A ordem de realização das traduções foi alternada aleatoriamente entre os sujeitos.

3. A esse respeito, ver Jakobsen, 2002.

4. Conforme os relatos retrospectivos dos sujeitos, os movimentos recursivos foram realizados no intuito de aprimorar o texto alvo.

5. Conferir Alves, Pagano e Silva (2009). Os autores usam os mesmos sujeitos desta pesquisa, porém, os dados são analisados a partir do rastreador ocular Tobbi Studio T60. 
6. Os textos consistem na introdução de um artigo acadêmico em inglês de 237 palavras e a introdução de um artigo acadêmico em português de 243 palavras, sobre anemia falciforme.

7. Ainda que alguns tradutores afirmem realizar mais traduções para o inglês, todos os sujeitos têm ampla prática em tradução direta e inversa.

8. A esse respeito, ver Rodrigues (2009).

9. Vale destacar que o tamanho dos segmentos está diretamente relacionado à quantidade de segmentos apresentada por cada tradutor, conforme observado em Rodrigues (2009): quanto mais segmentado o texto, i.e., quanto maior a quantidade de segmentos, menor será o tamanho dos mesmos.

10. A observação aponta para o problema do uso de textos correlatos, já que a primeira tradução exerce efeito facilitador quando da segunda. Dessa maneira, as próximas análises serão feitas a partir de textos de partidas que versem sobre diferentes assuntos, ainda que tenham nível de dificuldade semelhante, de acordo com a Teoria das Estruturas Retóricas (RST, MANN \& THOMPSON, 1987) .

11. A análise das pausas pode ser verificada na dissertação de mestrado de Ferreira (2010).

\section{Bibliografia}

ALVES, F. A triangulação como opção metodológica em pesquisas empírico-experimentais em tradução. In. PAGANO, A. (org.). Metodologias de pesquisa em tradução. Belo Horizonte: POSLIN/FALE/UFMG, 2001. p. 69-92.

. Tradução, Cognição e Contextualização: Triangulando a Interface Processo-Produto no Desempenho de Tradutores Novatos. D.E.L.T.A., v.19: Especial, 2003. p. 71-108. 
. Ritmo cognitivo, Metarreflexão e Experiência: parâmetros de análise processual no desempenho de tradutores novatos e experientes. In. PAGANO, A.; MAGALHÃES, C.; ALVES, F. (orgs.). Competência em Tradução: cognição e discurso. Belo Horizonte: Editora da UFMG, 2005. p. 109-153.

ALVES, F.; LIPARINI CAMPOS, T. Translation technology in time: investigating the impact of translation memory systems and time pressure on types of internal and external support. In: GÖPFERICH, S., JAKOBSEN, A. L., MEES, I. (orgs.). Copenhagen Studies in Language. Copenhagen: Samfunds Litteratur, v. 30, 2009. p. 191-218.

BARnStOnE, W. The Poetics of Translation. History, Theory, Practice. Yale University Press. 1993.

BUCHWEITZ, A; ALVES. F. Cognitive adaptation in translation: an interface between language direction, time, and recursiveness in target text production. Letras de Hoje, v. 41, 2006. p. 241-272.

DRAGSTED, B. Segmentation in translation: an empirical investigation of cognitive segmentation and effects of integrating a TM system into the translation process. 2004. 305 f. Tese (Doutorado) - Copenhagen Business School, Copenhagen, 2004.

FERREIRA, A. Direcionalidade em tradução: uma investigação do processamento cognitivo de tradutores profissionais em tradução direta e inversa no par linguístico inglês-português. Dissertação (Mestrado em Linguística Aplicada) - Faculdade de Letras, Universidade Federal de Minas Gerais, Belo Horizonte, 2010.

GARCÍA-YERBA, V. En torno a la traducción. Madrid: Gredos. 1983.

JAKOBSEN, A. L. Translation drafting by professional translators and by translation students. In: HANSEN, G. (Ed.). Empirical translation studies: process and product. Copenhagen: Samfundslitteratur, 2002. p. 191-204.

. Effects of think aloud on translation speed, revision and segmentation. In: ALVES, F. (Ed.). Triangulating translation: perspectives in process-oriented research. Amsterdan: John Benjamins, p. 69-95, 2003. 
KELLY, D.; MATÍN, A.; NOBS, M-L.; SÁNCHEZ, D.; WAY, C. Reflexiones en torno a algunos conceptos básicos. In: KELLY, D.; MATÍN, A.; NOBS, M-L.; SÁNCHEZ, D.; WAY, C. (Ed.). La direccionalidad en traducción e interpretación: perspectivas teóricas, profesionales y didácticas. Granada, 2003.

KROLL, J. F.; STEWART, El 1994. Category interference in translation and picture naming: Evidence for asymmetric connections between bilingual memory representations. Journal of Memory and Language, 33(2), 149-174.

LADMIRAL, J. R. Théorèmes pour la traduction. París: Didier, 1979.

LIPARINI CAMPOS, T. O efeito da pressão de tempo na realização de tarefas de tradução: uma análise processual sobre o desempenho de tradutores em formação. 2005. 191f. Dissertação (Mestrado em Linguística Aplicada) - Faculdade de Letras, Universidade Federal de Minas Gerais, Belo Horizonte, 2005.

. O efeito do uso de um sistema de memória de tradução e da pressão de tempo no processo cognitivo de tradutores profissionais. 2005. Tese (Doutorado em Linguística Aplicada) - Faculdade de Letras, Universidade Federal de Minas Gerais, Belo Horizonte, 2010.

LORENZO, M. P. La traducción a una lengua extranjera: uno de los muchos desafíos a la competencia traductora. In KELLY, D., MATÍN, A., NOBS, M-L., SÁNCHEZ, D., WAY, C. (Eds.). La direccionalidad en traducción e interpretación: perspectivas teóricas, profesionales y didáticas. Granada, 2003.

MILLER, N. A.; Kroll, J. F. (2002). Stroop effects in bilingual translation. Memory \& Cognition, 30, 614-628.

NEWMARK, P. A Textbook of Translation. Great Britain: Prentice Hall, 1987. p. 20.

POKORN, N, K. Translation into a non-mother tongue: Challenging the Traditional Axioms. University of Ljubljana, 2005.

RODRIGUES, R.R. Segmentação cognitiva e o Uso de Sistemas de Memória de Tradução: uma análise do processo tradutório de tradutores profissionais nos 
pares linguísticos alemão-português e inglês-português. Dissertação (Mestrado em Linguística Aplicada) - Faculdade de Letras, Universidade Federal de Minas Gerais, Belo Horizonte, 2009.

SCHILPEROORD, J. It's about time - Temporal aspects of cognitive processes in text production. Utrecht: USI \& C, 1996. 